\title{
Augment It Yourself
}

\author{
Aashay Jain \\ Sardar Patel Institute \\ of Technology \\ Mumbai, India
}

\author{
Anukrit Jain \\ Sardar Patel Institute \\ of Technology \\ Mumbai, India
}

\author{
Yash Parekh \\ Sardar Patel Institute \\ of Technology \\ Mumbai, India
}

\author{
Renuka Pawar \\ Sardar Patel Institute \\ of Technology \\ Mumbai, India
}

\begin{abstract}
AR represents a way of exhibiting your product on a much more personalized level. It has the potential to invade spaces like a retail, restaurant, or event venue, and access customized and exclusively created AR content for you, which makes it a personalized event. Brands look for interactive technology to give customers a more personalized experience. AR acts as an entry point and lets brands access new markets and new customer base. Minutely created virtual 3D models, coupled with AR will revolutionize the way companies understand customer needs and helps them continuously improve the marketing of their products and services, and identify new business models. A lot of companies into designing homes and workplaces let you imagine your empty rooms placed with their furniture and designs to let you enjoy a personalized experience. AR lets marketing personnel use less of those heavy non-interactive books and more of personalized AR. AR applications can directly access surfaces and augment the designs on them. AR / VR allows a company to get more of uniqueness and allows the consumer to have a different perspective about it. Hence companies are looking for a much generalized application to augment their products or services onto applications for a better experience.
\end{abstract}

\section{Keywords}

Augmented reality, 3D model, AR applications

\section{INTRODUCTION}

A. What is Augmented Reality?

It is a technology that superimposes a computer-generated image on a user's view of the real-world, thus providing a composite view.

B. How is it different from Virtual Reality?

Augmented reality adds digital elements to a live view often by using the camera on a device whereas Virtual reality is the use of computer technology to create a simulated environment.

\section{RELATED WORK}

Not much work has been done in the field of Augmented Reality and if we consider the field which we are working on is not much explored upon but will bring about a lot of resource saving and technological advancements.

A lot of papers suggest[6] that implementation of AR can help in the production line to all employees who want to understand the procedures and functioning of the machines and it also assists employee in regular maintenance of the machines.

Another study[5] suggests that augmented reality helps in the sales in marketing of large products as visualising them becomes easier and much more interactive for the user, as it is much more scale-able it helps in bridging the gap between the end user and the producer of the component to be augmented. Another study[8] suggests that augmented reality in the field

of education can help in the better visualization of the components of the subject under study, it also helps in getting a better view of the human body which gives them an experience of working on the actual human body.

Augmented Reality has been one of the least used technologies in India in spite of the tremendous use cases. This is because of the lack of open source libraries and a community. The research work on Augmented reality focuses more on the use cases than on the implementation.

However, there is a good amount of research work when it comes to implementing Augmented Reality in the field of gaming. While Augmented Reality has been proven to be a great tool for the gaming industry, the implementation of the problem solving aspect of the same is neglected.

\section{METHODOLOGY}

The main challenge for us in developing a generalized crossplatform application was to get a work around of the tagging feature in which the user can select a specific area of the 3D model and add tags and information to those components which consists of video, document and IOT values tab.

Augmenting a tag to any part of a 3D model means the need to carefully understand the mesh and the structure of the model. Our scripts automate this process for us by understanding the structure and rendering the features that the user wants to have. The segregation feature implementation was also quite a tedious task as in this the $\mathrm{x}, \mathrm{y}$ and $\mathrm{z}$ coordinates of each component had to be obtained and then each object had to be displaced either in the forward or backward direction depending on what is the position of that particular component. The scripts ensure that the child objects of any object moves in the same direction resulting in a user friendly segregation. Adding rotation to the object is also a little tricky in terms of determining the local position of each object in the model and rotating them all together in the world space without tampering any of the other features. With this, we also implemented drag- ging of the embedded data like YouTube screen, documents and images.

\section{A. Use Case Diagram}

The Use Case diagram outlines the different types of functionalities and rights given to different types of users. The basic users have only the authority to use the end application which has the augmented 3D model, defined hotspots on that model and they can enjoy the visual experience of that particular model with video information and attached documents. 


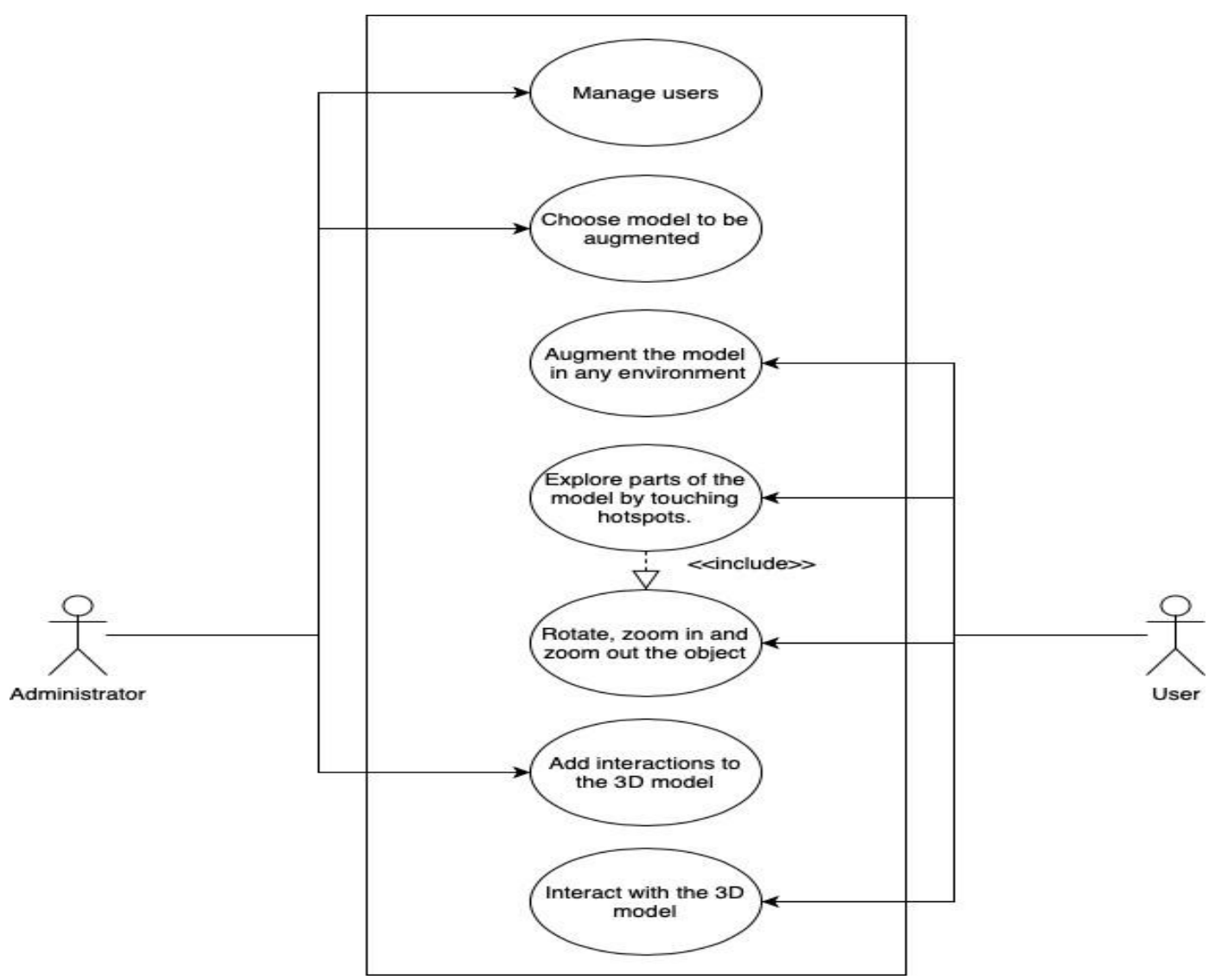

Fig. 1. Use Case Diagram

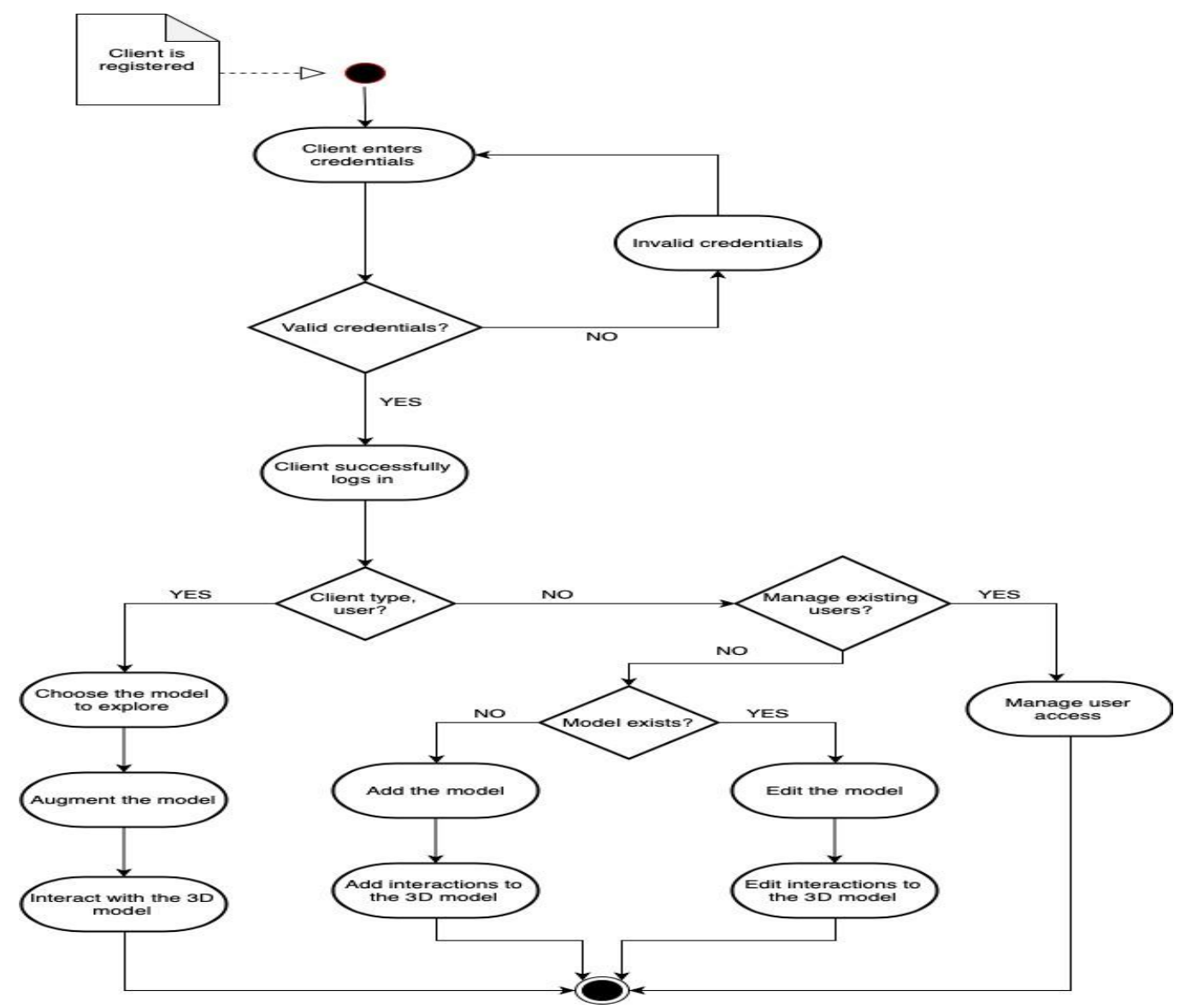

Fig. 2. Activity Diagram 


\section{B. Activity Diagram}

The activity diagram shows the flow of how the application functions and what are the different queries that are encountered during the course of the functioning of the application for different types of users which are the administrator and the end-user of the product.

The application lets the user select whether the account is a user or an administrator account and then gives them the required functionality whether to view and interact with the object or just to add or edit the new or existing model respectively.

\section{Block diagram}

The block diagram given below shows the different components that function in our system to make the augmentation functionality to work.

It shows the flow of the application from the start how it starts at the user's device and how the internal components react to the image marker when it is scanned from the camera and all the data is retrieved from the different databases which have all the stored data.

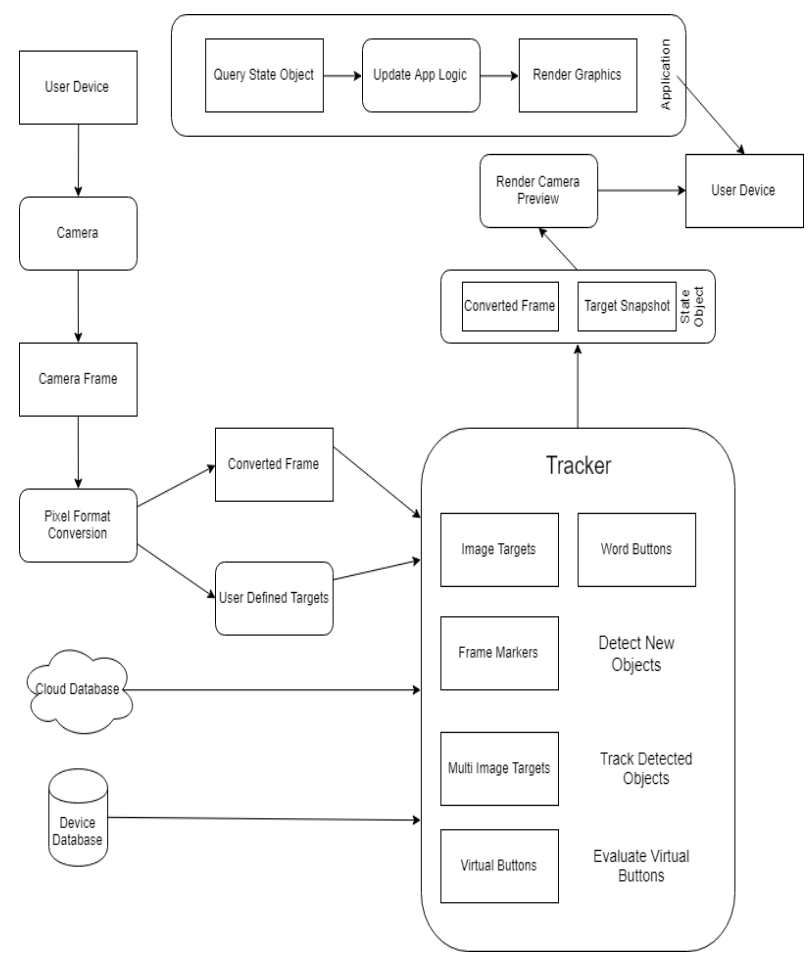

Fig. 3. Block Diagram

\section{RESULTS AND IMPLEMENTATIONS}

\section{A. Results}

Augment It Yourself lets users define hot-spots on the 3D model which are defined by the user using drag feature. These hot-spots let user add and modify data about the selected area. Also a code has been deployed which lets the users interact with the model by using zoom in, zoom out and rotate.Image processing scans the image marker to augment the 3D model into the real world space. Clicking a button on the user interface lets the user to see even the smallest part of the $3 \mathrm{D}$ model.

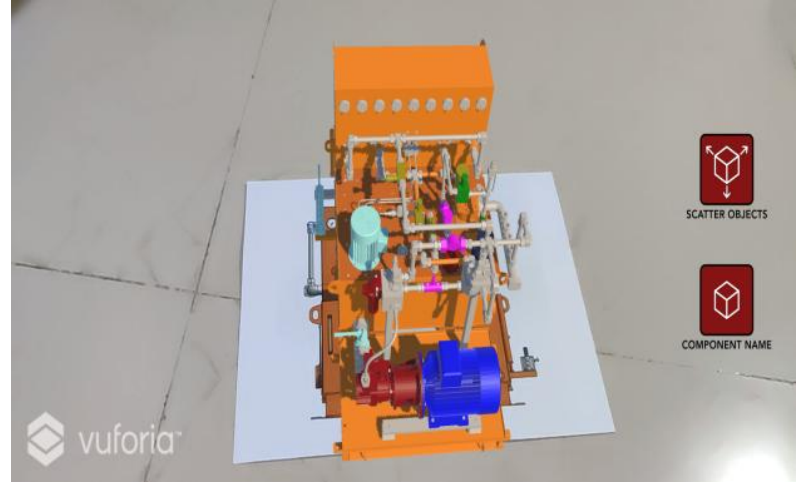

Fig. 4. 3D Model Augmented on Marker

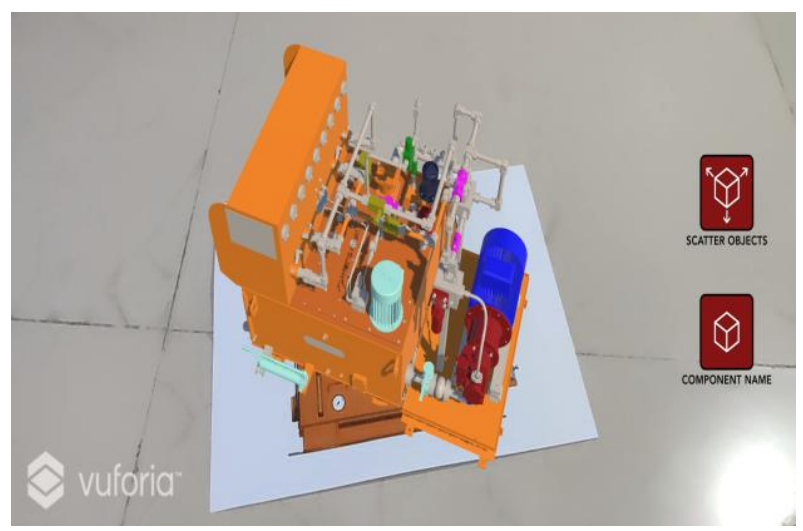

Fig. 5. Rotation Feature of model

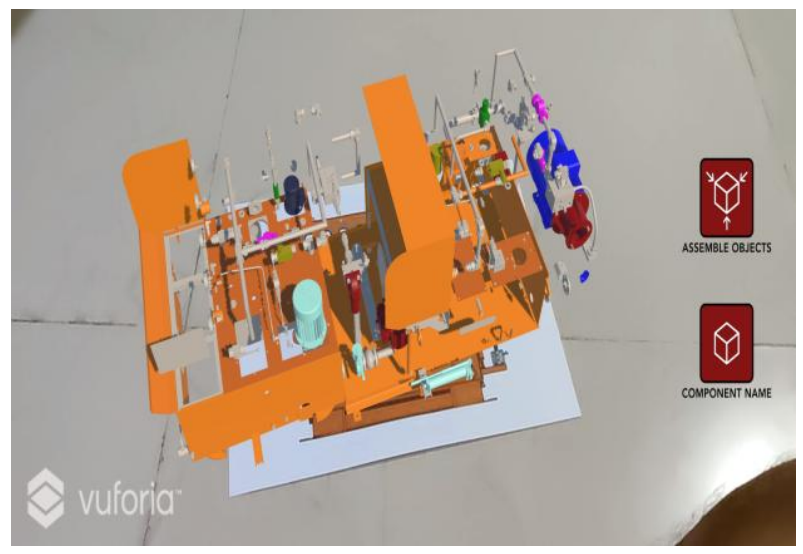

Fig. 6. Segregation of the 3D Model

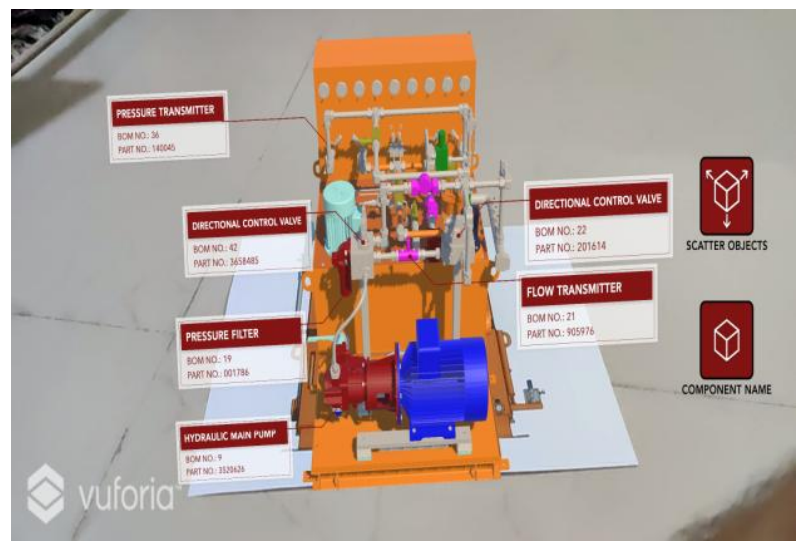

Fig. 7. Component Names come up when Component Name tab is clicked 


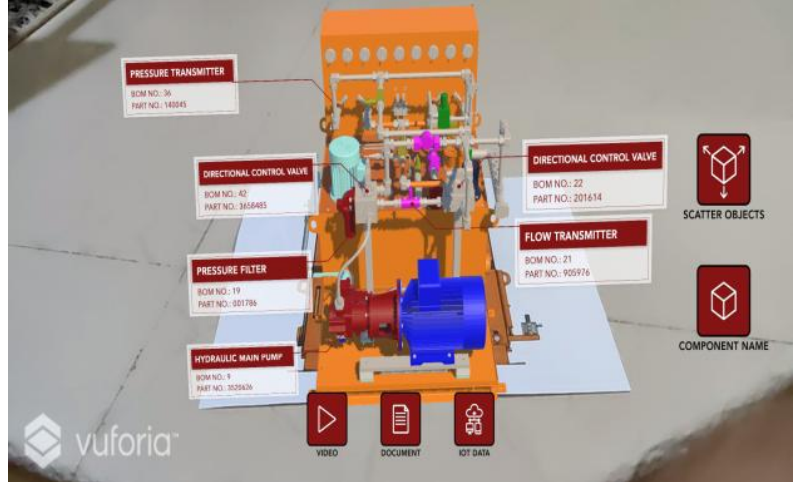

Fig. 8. New button panel comes up when any panel is clicked

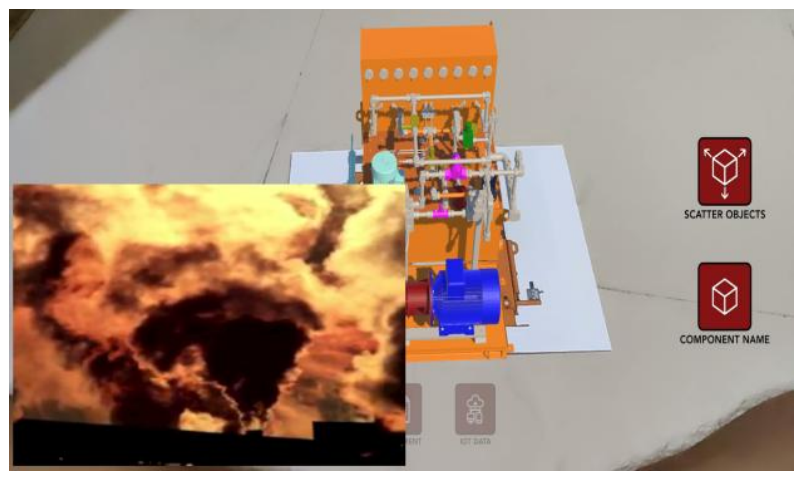

Fig. 9. Youtube Player comes up when Video Button is clicked

\section{B. Social Impact}

Augmented Reality is a way to visualise things without the need of physically having to look at it. The number of resources saved in showing a product with the help of

Augmented Reality is large. In medicine, this technology can be used to understand the human anatomy in the best possible way which increases the efficiency in learning medicine and visualizing an operation before actually performing it. This can be used to understand various health issues. In terms of supply chain, AR gives consumer the exact idea about his purchases and educates him about the environmental costs of the product.

\section{LIMITATIONS AND FUTURE WORK}

The current limitations with Augment It Yourself is that the 3D model had to be provided to the developers to be augmented into the application. The current software also augments the model only in the presence of a predefined image marker, which has been added to the software by the developer.

The application has to be downloaded to the device using app store or play store for the iOS or Android devices respectively using a valid data connection.

The future efforts would be to give the user the freedom of uploading the $3 \mathrm{D}$ model to the software themselves and also there would be no need of an image marker to augment the objects. The application will itself scan the surface to find pixels and form a mesh to augment the 3D model.

\section{REFERENCES}

[1] Gang Li, Yue Liu, Evaluation of labelling layout methods in augmented reality, 18 March 2017: https://ieeexplore.ieee.org/document/7892321

[2] Mario Lorenz, Sebastian Knopp, Industrial Augmented Reality: Require- ments for an Augmented Reality Maintenance Worker Support System, 16 October 2018: https://ieeexplore.ieee.org/document/8699183

[3] Vicenzo Abichequer Sangalli ; Thomas Volpato de Oliveira, Sculp- tAR: An augmented reality interaction system,18March2017:https://ieeexplore.ieee.org/docume $\mathrm{nt} / 7893371$

[4] Jun He Peng Han,The research and application of the augmented reality technology, 15 December 2017: https://ieeexplore.ieee.org/document/8284781

[5] Dennise Adrianto Monica Hidajat, Augmented reality using Vuforia for marketing residence, 15 December 2017: https://ieeexplore.ieee.org/document/8052642

[6] scarBlancoNovoa,TiagoM.FernndezCarams, APracticalE valuationofCommercialIndustrialAugmentedRealitySyste $\mathrm{ms}$ in an Industry 4.0 Shipyard, 05February2018: https://ieeexplore.ieee.org/document/8281493

[7] Tobias Blum ; Valerie Kleeberger ; Christoph Bichlmeier ; Nassir Navab, mirracle: An augmented reality magic mirror system for anatomy edu- cation, 4-8 March 2012: https://ieeexplore.ieee.org/document/6180909

[8] Sidharth Sunil ; Smitha Sunil Kumaran Nair, An Educational Aug- mented Reality App To Facilitate Learning Experience, 6-7 Sept. 2017: https://ieeexplore.ieee.org/document/8079771

[9] Vinh T. Nguyen ; Tommy Dang, Setting up Virtual Reality and Augmented Reality Learning Environment in Unity,913Oct.2017:https://ieeexplore.ieee.org/document/ 8088512

[10] Ryosuke Umeda ; Mohamed Atef Seif ; Hiroki Higa ; Yukio Kuniyoshi, A medical training system using augmented reality, 24-26 Nov. 2017: https://ieeexplore.ieee.org/document/8279706

[11] Julie Carmigniani, Borko Furht, Marco Anisetti, Paolo Ceravolo, Ernesto Damiani and Misa Ivkovic; "Augmented Reality technologies, system and applications"; Springer 2010

[12] J. Michale Specter, M.David Merill, Jan Elen and, MJ. Bishop; "Hand- book of Research on Education Communications and Technology"; Springer 2014

[13] Yan Fei Gao, Heng-you Wang and, Xiao-Nang Bian; "Marker tracking for video-based augmented reality"; IEEE 2016

[14] Sanni Siltanen; "Theory and application of marker-based augmented reality"; VTT 2012

[15] Fuguo Peng and, Jing Zhai; "A Mobile Augmented Reality System for Exhibition Hall Based on Vuforia"; IEEE 2017 